\title{
Development of real-time photoacoustic microscopy
}

Lidai Wang, Konstantin Maslov, Junjie Yao, Li Li, Lihong V. Wang

Lidai Wang, Konstantin Maslov, Junjie Yao, Li Li, Lihong V. Wang, "Development of real-time photoacoustic microscopy," Proc. SPIE 7899, Photons Plus Ultrasound: Imaging and Sensing 2011, 78990R (17 February 2011); doi: 10.1117/12.875415

SPE. Event: SPIE BiOS, 2011, San Francisco, California, United States 


\title{
Development of real-time photoacoustic microscopy
}

\author{
Lidai Wang, Konstantin Maslov, Junjie Yao, Li Li, Lihong V. Wang* \\ Department of Biomedical Engineering, Optical Imaging Laboratory, \\ Washington University in St. Louis, \\ Campus Box 1097, One Brookings Drive, St. Louis, Missouri 63130-4899, USA
}

\begin{abstract}
Photoacoustic tomography detecting ultrasound signals generated from photon absorption provides optical absorption contrast in vivo for structural, functional and molecular imaging. Although photoacoustic tomography technology has grown fast in recent years, real-time photoacoustic imaging with cellular spatial resolution are still strongly demanded. We developed a photoacoustic microscopy which has video-rate imaging capability with cellular spatial resolution. The system consists of a single-element focused ultrasound transducer, a fiber-based light-delivery subsystem, a voice-coil translation stage, a motion controller, and a data acquisition subsystem. A compact cube is employed to split optical and acoustic beams. The mass of the entire scanning photoacoustic probe is less than 40 grams, which minimizes potential vibrations and inertial effects, therefore, makes it capable to scan fast. The imaging system is capable of acquiring 20 cross-sectional (B-scan) images per second over $9 \mathrm{~mm}$, and up to $40 \mathrm{~B}$-scan images per second over $1 \mathrm{~mm}$. Focused laser beams provide a lateral resolution of five microns. Confocal deployment of optical and acoustic focuses provides higher SNR than optical scanning approach. Micron-sized carbon particles flowing in silicone tubing and in vivo blood flows were imaged in video-rate, which demonstrated the capability to image highly dynamic biological processes in vivo with cellular resolution. This real-time high-resolution photoacoustic imaging system provides a promising approach for various in vivo imaging and quantitative studies.
\end{abstract}

Keywords: Photoacoustic tomography, photoacoustic microscopy, fast scanning, real time, optical resolution, in vivo

\section{INTRODUCTION}

Photoacoustic imaging technology detects ultrasonic signals generated from photon absorption, which provides unique optical absorption contrast in vivo for structural, functional and molecular imaging [1-8]. Photoacoustic tomography has two major modes: computed tomography and focused scanning tomography (also referred as photoacoustic microscopy or PAM). Computed photoacoustic tomography employs circular scanning [1,9] or array based ultrasound transducers $[10,11]$ to detect photoacoustic signals. Array based ultrasound transducers allowed for high frame rates up to $50 \mathrm{~Hz}$ [10]. Lateral resolution of this high speed photoacoustic system was determined by acoustic parameters (about 80 microns). In focused scanning photoacoustic tomography, a single focused ultrasound transducer is mechanically scanned over a two dimensional plane to acquire a three dimensional (volumetric) image. At each scanned position, the acoustic time-of-flight provides depth information; the acoustic focusing (AR-PAM) [4] or optical focusing (OR-PAM) [12], depending on which focuses tighter, yields lateral resolution. Compared with computed tomography, the focused scanning tomography has simpler structure and lower cost. Moreover, the spatial resolution of PAM can be scaled to image objects from sub-cellular organelles to organs [13]. OR-PAM can provide high lateral resolution from several microns to sub microns $[12,14]$. However, most PAM systems utilized precision ball screw or lead screw mechanical scanning mechanisms, which were inherently difficult to achieve high scanning speed. A high speed voice-coil stage has been used to improve scanning speed in an AR-PAM system, but only slow photoacoustic imaging was demonstrated [15]. Other PAM modality improved scanning speed by utilizing optical scanning and weak acoustic focusing [16]; however, the weak acoustic focusing and non-confocal deployment of optical and acoustic beams decreased signal-tonoise ratio (SNR), which would decrease imaging quality and affect quantitative functional imaging.

*lhwang@seas.wustl.edu; phone 1314 935-6152; fax 1314 935-7448; oilab.seas.wustl.edu

Photons Plus Ultrasound: Imaging and Sensing 2011, edited by Alexander A. Oraevsky, Lihong V. Wang, Proc. of SPIE Vol. 7899, 78990R - (C) 2011 SPIE · CCC code: 1605-7422/11/\$18 - doi: 10.1117/12.875415 
To date, fast scanning photoacoustic microscopy that has high spatial resolution and high SNR is strongly demanded to image dynamic biological processes in vivo. For example, noninvasive real-time imaging of blood flow in microvasculature would open a new window to physiology studies. Although Doppler [17] and M-mode [18] methods have been utilized for photoacoustic flow measurements, both methods only measure flow velocity projected onto one axis: either along or perpendicular to the ultrasound axis. By contrast, fast scanning imaging has potential to measure flow speed in a two-dimensional field. On the other hand, fast scanning speed can sufficiently reduce the time to acquire a volumetric image so that dynamic processes can be imaged and motion artifacts can be mitigated. These are of much importance to the future clinical application of photoacoustic imaging.

We developed a novel OR-PAM system that provides video-rate B-scan imaging with high-SNR. The system utilizes a voice-coil linear translation stage to achieve high-speed scanning. Tight optical focusing provides a high lateral resolution of $5 \mu \mathrm{m}$. Because high-speed mechanical scanning can introduce oscillating inertial force and vibrations, which would decrease lateral resolutions of OR-PAM systems, light weight design of the scanning probe and suppressing vibration is the key to developing a fast-scanning high-resolution PAM system. The scanning photoacoustic probe in this system is designed to be lightweight, which minimizes inertial effects and vibrations, therefore, making it capable to scan in video rate. Micron-sized carbon particles flowing in silicone tubing and blood flows in veins were imaged in video-rate, which demonstrates real-time imaging capability and high spatial resolution,

\section{SYSTEM DESIGN AND CHARACTERIZATIONS}

\subsection{System design}

As shown in Figure 1, a pulsed laser, which is a dye laser pumped by a Nd:YLF laser, delivers light to the photoacoustic probe through a single mode optical fiber. The highest pulse repetition rate is $4 \mathrm{kHz}$. The laser pulse duration is shorter than $10 \mathrm{~ns}$. The laser pulse beam is focused by a pair of optical lenses, and then reflected on an aluminum-coated prism. This prism and another uncoated prism are tightly bonded together to create an optical-acoustic beam splitter. Since the aluminum-coated layer has an ultrasound impedance close to glass, this beam splitter can transmit ultrasound without significant loss. Reflecting optical beams requires smaller sized components than reflecting acoustic beams. This beam splitter design is more compact and lighter than reflecting acoustic beams and transmitting optical beams. The laser pulse energy delivered on to the object may be varied from several tens of $\mathrm{nJ}$ to over $100 \mathrm{~nJ}$ depending on the imaging application. A high-frequency ultrasound transducer (central frequency, $75 \mathrm{MHz}, \mathrm{V} 2022 \mathrm{BC}$, Olympus NDT) is employed to detect photoacoustic signals. The ultrasound transducer focuses onto the same spot with the optical beam through the beam splitter and an acoustic concave lens (aperture, $6 \mathrm{~mm}$; NA in water, 0.5). The tight acoustic focus and optical-acoustic confocal deployment provides higher SNR compared with optical scanning photoacoustic microscopy systems. The optical and acoustic components are attached to a base plate to form a photoacoustic probe. The photoacoustic probe weighs less than 40 grams, which minimized inertial force and potential vibrations during fast scanning.

The photoacoustic probe is mounted on a voice-coil linear translation stage (VCS-1010 Equipment Solutions, Sunnyvale
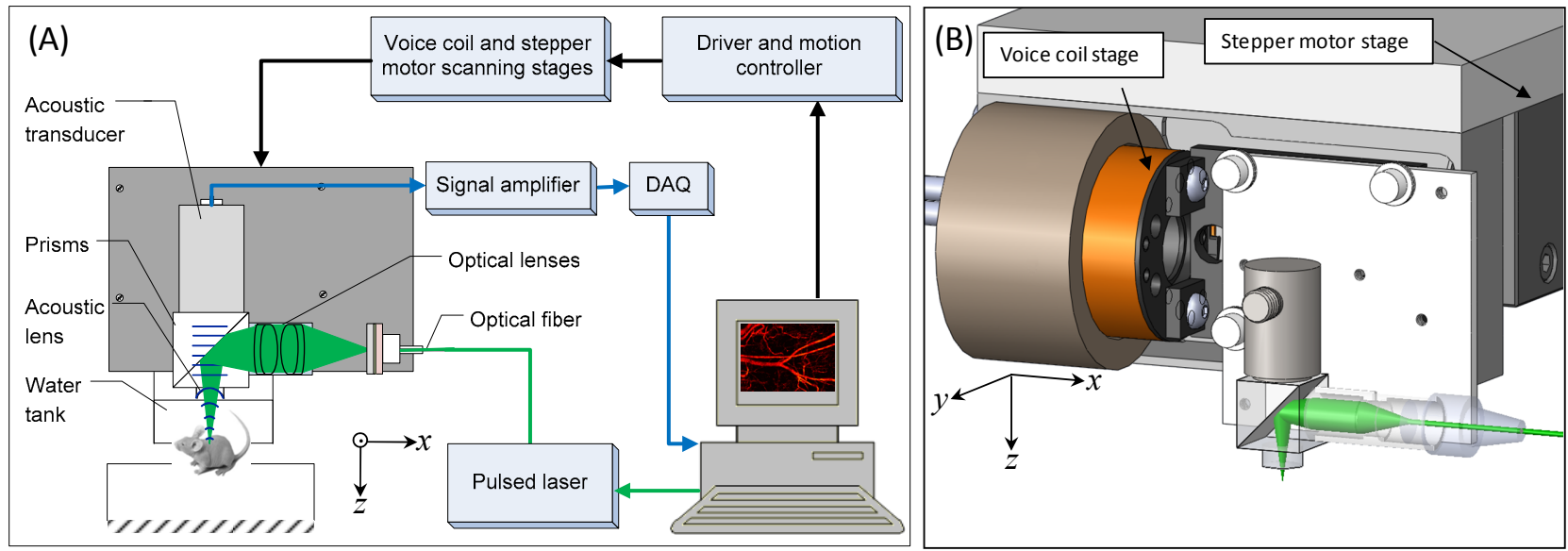

Figure 1. (A) Schematic and (B) three-dimensional model of real-time photoacoustic system. 
CA, USA), which creates a fast-scanning axis ( $x$-axis). The voice-coil stage has high force-to-mass ratio and small friction, therefore low vibration during high-speed scanning. A proportional-integral-derivative (PID) motion controller is employed to drive the voice-coil stage to scan at a predefined B-scan rate. A precise stepper motor linear stage (PLS85, Micos, Eschbach, Germany) is utilized to create a slow-scanning axis ( $y$-axis). Photoacoustic signals are amplified by two low-noise amplifiers (ZFL-500LN+, Mini-circuits, NY, USA), and then acquired by a high-speed digitizer (DAQ) (ATS9350, Alazar tech Inc., Pointe-Claire, QC, Canada), using a sampling rate of $500 \mathrm{MHz}$. A motion control card (PCI-6251, National Instrument, Austin, TX, USA) generates scanning motion commands and triggers both the pulsed laser and the DAQ. At each scanning position, the scanning control card sends out a digital pulse to trigger the laser and the DAQ simultaneously. The DAQ starts to acquire photoacoustic signals at $500 \mathrm{MHz}$ with a trigger delay of several micro-seconds. To minimize the jitter of the photoacoustic signal, base clocks of the DAQ card and the motion control card are synchronized through a $10 \mathrm{MHz}$ reference clock. The time of flight of the photoacoustic signal carries depth information. Each scan along the $x$-axis provides one B-scan image. Multiple B-scan images generate a threedimensional image. The photoacoustic imaging system is capable of scanning at $20 \mathrm{~Hz}$ B-scan frame rate over $9 \mathrm{~mm}$, and up to $40 \mathrm{~Hz}$ over $1 \mathrm{~mm}$. The fast scan speed shortens the time to acquire a volumetric image by tens of folds. The imaging system can work in a repetitive B-scan mode to image highly dynamic biological processes in real time, which would be useful for in vivo quantitative functional imaging.

\subsection{System characterizations}

The lateral resolution of the PAM system was quantified by imaging a carbon fiber in optically non-scattering medium. A maximum amplitude projection (MAP) image of the carbon fiber and the signal profile along the dash line is shown in Fig. 2 (A). The measured full width at half maximum (FWHM) of the carbon fiber is 8.2 microns. Compared with 6
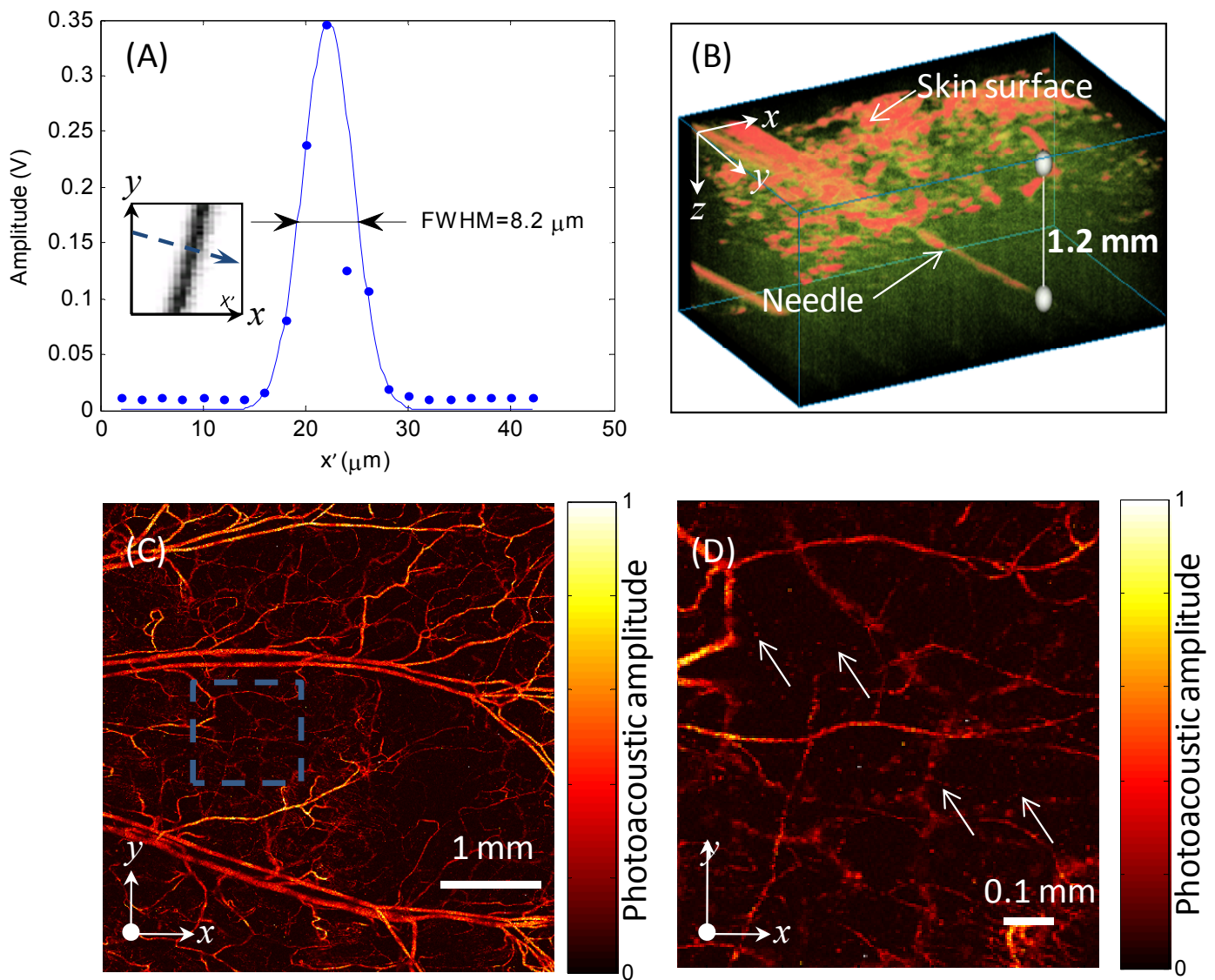

Figure 2. (A) Lateral resolution test on a 6- $\mu \mathrm{m}$ carbon fiber (B) Test of penetration depth by imaging a needle inserted in mouse tissue. (C) In vivo maximum amplitude projection (MAP) image of a mouse ear vasculature. (D) Close-up image of the region enclosed by the dashed lines in (C), arrows denote single red blood cells (RBCs). 
microns nominal diameter of the carbon fiber, the measured FWHM is 2.2 microns wider. Considering the focused laser beam might slightly diffuse in biological tissue, we claim the lateral resolution as 5 microns. This result agrees with another OR-PAM [12], which has similar design in term of optical and acoustic focusing. The axial resolution was determined by the system bandwidth and the sound speed in tissue $(\sim 1.5 \mu \mathrm{s} / \mathrm{ns})$. The bandwidth of this PAM system is $100 \mathrm{MHz}$ in receiving-only mode, which provides an axial resolution of 15 microns. The penetration depth of the PAM was measured by imaging a needle (diameter 240 microns) inserted into mouse tissue. A volumetric image is shown in Fig. 2 (B). The needle is visible below $1.2 \mathrm{~mm}$ depth into the tissue. Fig. 2 (C) shows a micro-vasculature image of a nude mouse ear in vivo at an optical wavelength of $570 \mathrm{~nm}$. Fig. 2 (D) is a close-up image of the dashed region-ofinterest area in Fig. 2 (C). Single RBCs in capillaries were clearly resolved, which demonstrates the optical resolution.

The SNR of the imaging system was measured by imaging a graphite sample (99.995\%, Sigma-Aldrich Co., Saint Louis, MO, USA). The laser energy used in this test was $40 \mathrm{~nJ}$ per pulse. The averaged SNR was measured to be $45 \mathrm{~dB}$. This SNR is higher than reported SNR results of optical scanning PAMs tested under the similar experimental conditions.

\section{REAL-TIME FLOW IMAGING}

Carbon particle flows were imaged in real time in repetitive B-scan mode. Average diameter of the carbon particles (Sigma-Aldrich, St. Louis, Missouri, USA) was $6 \mu \mathrm{m}$. The carbon particles were suspended in water (concentration: $0.1 \%$ ) and injected into a straight transparent rubber tube with an inner diameter of $250 \mu \mathrm{m}$ (Dow corning, Midland, Michigan, USA). A syringe pump (BSP-99M; Braintree Scientific, Braintree, Massachusetts, USA) was used to control the flow speed. The B-scan direction is parallel with the tube. B-scan images were acquired repetitively at $20 \mathrm{~Hz}$. The laser wavelength was set to $572 \mathrm{~nm}$. Figure 3 (A) shows a representative B-scan image of suspended carbon particles and a layer of particles depositing at the bottom of the tube. The high-resolution real-time B-scan images provided rich information, such as the flow direction and flow speed distribution along the radial direction of the tube.

To calculate flow speed at one $z$-axis position, the photoacoustic signal was plotted in $x$-axis and time axis plane. Trajectories of single carbon particles were shown in Figure 3 (B). The average slop of the trajectories can be used to compute the flow speed. The flow speed $v$ at depth $z$ can be written as

$$
v(z)=k \frac{\Delta x}{\Delta t+\frac{\Delta x}{v_{s}}}=k \frac{1}{1+\frac{\Delta x / \Delta t}{v_{s}}} \frac{\Delta x}{\Delta t}
$$

where $\mathrm{k}$ is a constant coefficient determined by scanning step size and B-scan rate, $v_{s}$ is the known scanning speed, $\Delta x$ and $\Delta t$ are directly determined from Figure 3 (B). When the scanning speed is much higher than the flow speed, which is the case here, the term $\frac{\Delta x / \Delta t}{v_{s}}$ may be neglected. The flow speed may be approximated with

$$
v(z)=k \frac{\Delta x}{\Delta t}
$$

Figure 3 (C) shows the flow speed distribution on radial direction of the tube. An approximate parabolic distribution is obtained. 

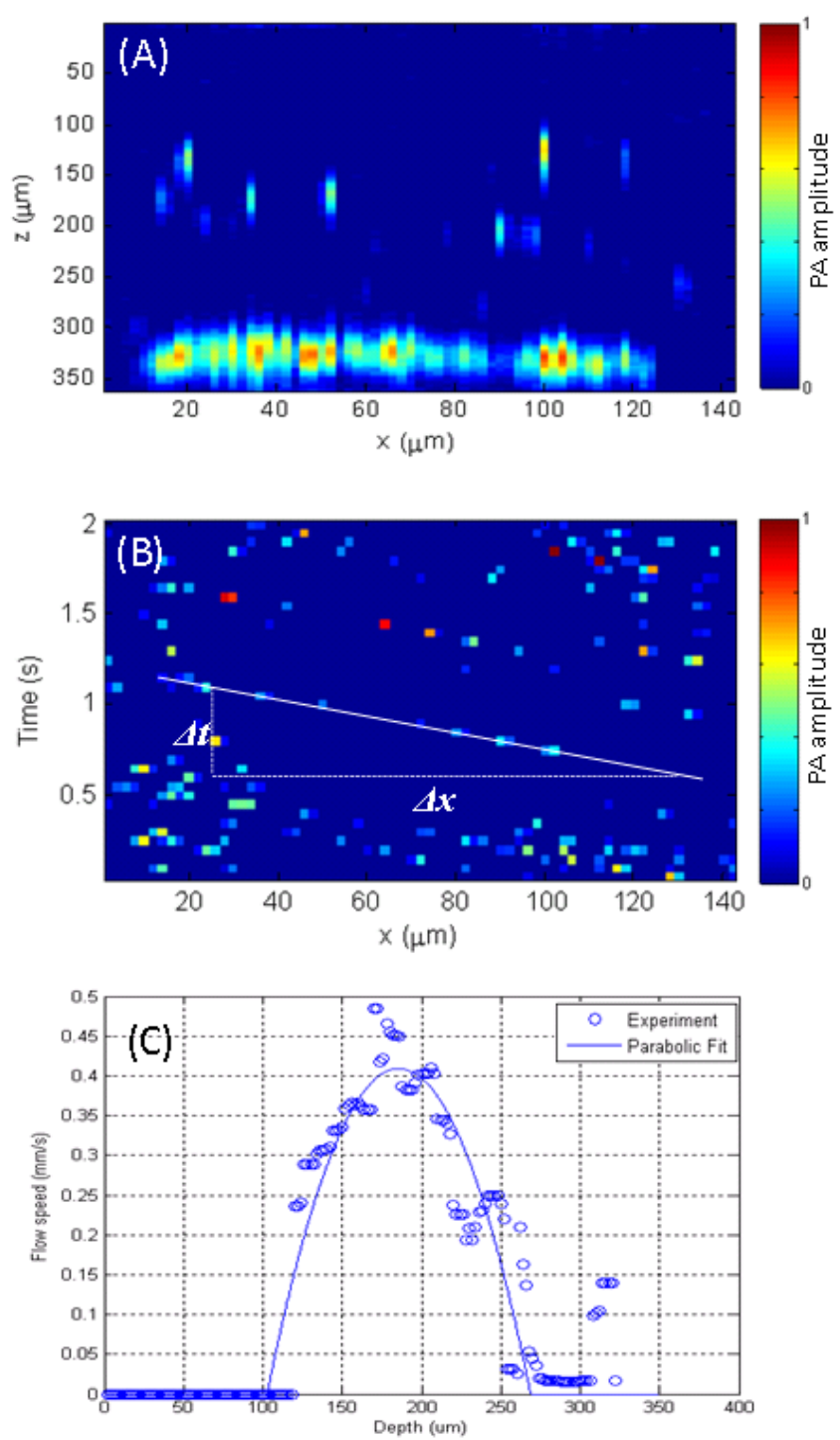

Figure 3. (A) Representative B-scan Image (B) carbon particles distribution at $x$-axis and time axis.

(C) flow distribution on $\mathrm{z}$-axis direction

Blood flows in vein were imaged in vivo in $40 \mathrm{~Hz}$ repetitive B-scan mode with a $40 \mathrm{kHz}$ pulse laser (532 nm). Figure 4 (A)-(E) show 5 representative B-scan images of blood flow in a vein, where different concentrations of RBCs were clearly imaged. Figure 4 (F) plots the MAP of each B-scan image on $x$-axis and time axis. Computing the slope of RBC concentrations changing with time provide the flow distribution on vein vessel radial direction. Note that the flow measurement does not require the scanning direction to be parallel with the flow direction. As long as the scanning direction is not close to the perpendicular direction of the flow, we can always compensate for the measured flow speed with an angle-related coefficient. Flows perpendicular to the original scanning direction can be readily measured by rotating the scanning direction by 90 degrees.

All experimental animal procedures were carried out in conformance with the laboratory animal protocol approved by the Animal Studies Committee of Washington University in St. Louis. 

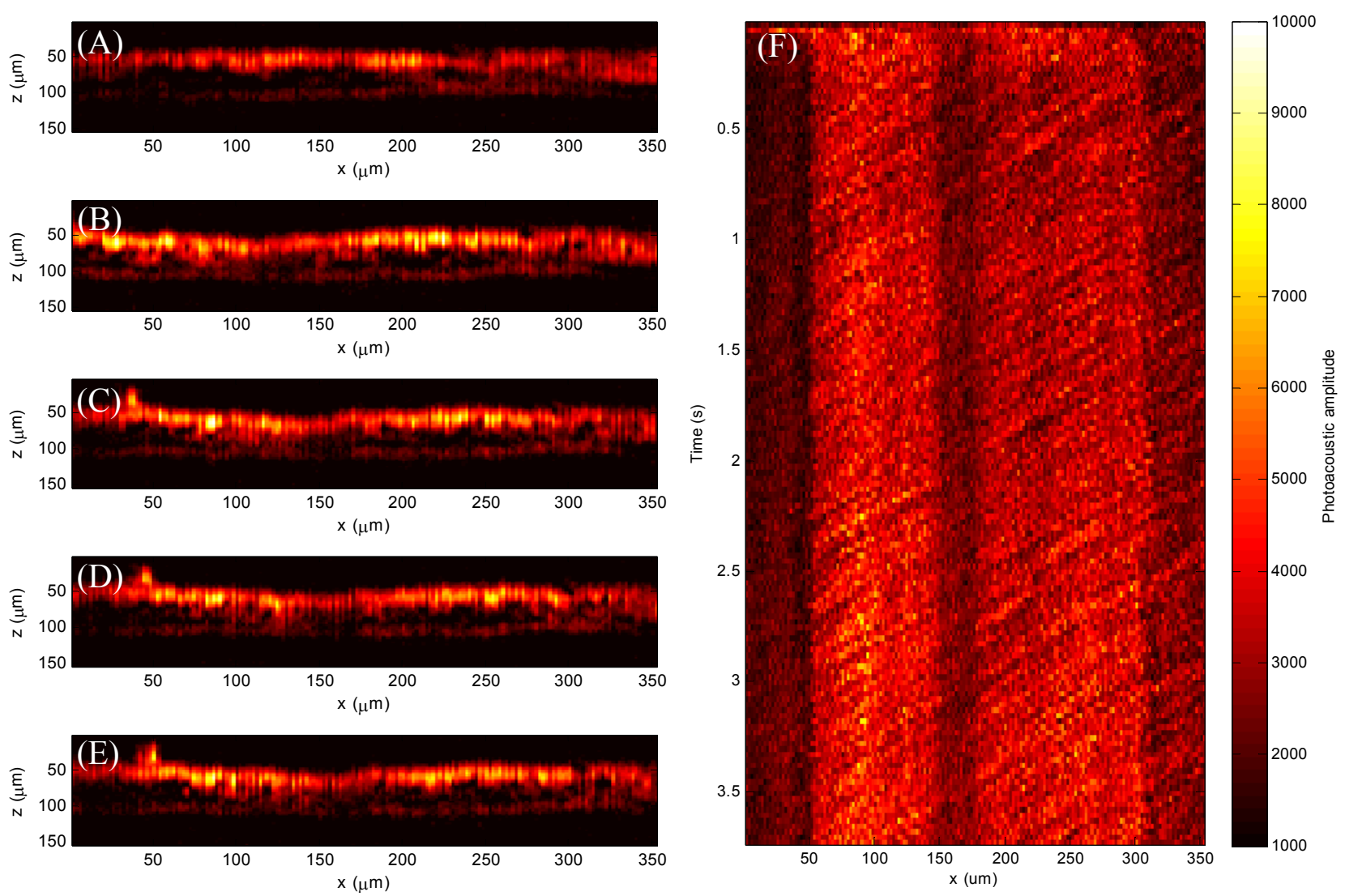

Figure 4. (A)-(E) 5 consequential B-scan images, (F) MAP of multiple B-scan images on $x$-axis and time axis

\section{CONCLUSION}

The real-time OR-PAM capable of scanning up to $40 \mathrm{~Hz}$ B-scan frame rate can improve the imaging speed by tens of folds, reduce motion artifacts, and image highly dynamic biological processes with micron resolution. This is a solid step towards future clinical application of PAM. High SNR is the key to do accurate quantitative study, e.g., oxygen saturation. The real-time PAM maintains confocal deployment of optical and acoustic focus, which provides high SNR than only optical scanning.

\section{ACKNOWLEDGEMENT}

We thank Song Hu, Dakang Yao, Liang Song, and Christopher Favazza for helpful discussions. This work was sponsored in part by National Institutes of Health grants R01 EB000712, R01 EB008085, R01 CA134539, U54 CA136398, R01 EB010049, and 5P60 DK02057933. L. V. Wang has a financial interest in Microphotoacoustics, Inc. and Endra, Inc., which, however, did not support this work.

\section{REFERENCES}

[1] X. Wang, Y. Pang, G. Ku, X. Xie, G. Stoica, and L. V. Wang, "Noninvasive laser-induced photoacoustic tomography for structural and functional in vivo imaging of the brain," Nature Biotechnol. 21, 803-806 (2003)

[2] J. A. Copland, M. Eghtedari, V. L. Popov, N. Kotov, N. Mamedova, M. Motamedi and A. A. Oraevsky, "Bioconjugated gold nanoparticles as a molecular based contrast agent: Implications for imaging of deep tumors using optoacoustic tomography," Mol. Imaging Biol. 6, 341-349 (2004) 
[3] R. I. Siphanto, K. K. Thumma, R. G. M. Kolkman, T. G. van Leeuwen, F. F. M. de Mul, J. W. van Neck, L. N. A. van Adrichem, and W. Steenbergen, "Serial noninvasive photoacoustic imaging of neovascularization in tumor angiogenesis," Opt. Express 13, 89-95 (2005)

[4] H. F Zhang, K. Maslov, G. Stoica and L. V Wang, "Functional photoacoustic microscopy for high-resolution and noninvasive in vivo imaging" Nature Biotechnology 24, 848 - 851 (2006)

[5] L. Li,, R. J. Zemp, G. Lungu, G. Stoica, and L. V. Wang, "Photoacoustic imaging of lacZ gene expression in vivo," J. Biomed. Opt. 12, 020504 (2007)

[6] S. Yang, D. Xing, L. Xiang, and Y. Lao, "Functional imaging of cerebrovascular activities in small animals using high-resolution photoacoustic tomography," Med. Phys. 34, 3294-3301 (2007)

[7] S. Mallidi, T. Larson, J. Aaron, K. Sokolov, and S. Emelianov, "Molecular specific optoacoustic imaging with plasmonic nanoparticles," Opt. Express 15, 6583-6588 (2007)

[8] M. Pramanik, K. H. Song, M. Swierczewska, D. Green, B. Sitharaman and L. V. Wang, "In vivo carbon nanotubeenhanced non-invasive photoacoustic mapping of the sentinel lymph node," Phys. Med. Biol. 54, 3291 (2009)

[9] H. Brecht, R. Su, M. Fronheiser, S. A. Ermilov, A. Conjusteau, A. A. Oraevsky, "Whole-body three-dimensional optoacoustic tomography system for small animals," J. Biomed. Opt. 14, 064007 (2009)

[10] R. Zemp, L. Song, R. Bitton, K.K. Shung, and L. V. Wang, "Realtime photoacoustic microscopy in vivo with a 30MHz ultrasound array transducer," Optics Express 16, Issue 11, pp. 7915-7928 (2008)

[11] J. Gamelin, A. Maurudis, A. Aguirre, F. Huang, P. Guo, L. V. Wang, and Q. Zhu, "A real-time photoacoustic tomography system for small animals," Optics Express, 17, Issue 13, pp. 10489-10498 (2009)

[12] K. Maslov, H. F. Zhang, S, Hu, and L. V. Wang, "Optical-resolution photoacoustic microscopy for in vivo imaging of single capillaries," Optics Letters, 33, Issue 9, pp. 929-931 (2008)

[13] L. V. Wang, "Multiscale photoacoustic microscopy and computed tomography," Nature Photonics 3, 503 - 509 (2009).

[14]K. Maslov, G. Ku, and L. V. Wang, "Photoacoustic microscopy with submicron resolution," Proc. SPIE 7564, $75640 \mathrm{~W}(2010)$

[15] T. Harrison, J. C. Ranasinghesagara, H. Lu, K. Mathewson, A. Walsh, and R. J. Zemp, "Combined photoacoustic and ultrasound biomicroscopy," Optics Express, 17, Issue 24, pp. 22041-22046 (2009)

[16]Z. Xie, S. Jiao, H. F. Zhang, and C. A. Puliafito, "Laser-scanning optical-resolution photoacoustic microscopy," Optics Letters, 34, Issue 12, pp. 1771-1773 (2009)

[17] H. Fang, K. Maslov, and L. V. Wang, "Photoacoustic Doppler flow measurement in optically scattering media," Appl. Phys. Lett. 91, 264103 (2007)

[18]H. Fang and L. V. Wang, "M-mode photoacoustic particle flow imaging," Optics Letters 34, Issue 5, pp. 671-673 (2009) 